\title{
A multicenter prospective study on the efficacy and safety of denosumab in gastrointestinal cancer patients receiving short-term periodic steroid premedication for prevention of chemotherapy- induced nausea and vomiting (ESPRESSO- 02/HGCSG1602).
}

Michio Nakamura ( $\nabla$ michio.nakamura@icloud.com )

Sapporo City General Hospital https://orcid.org/0000-0002-1509-9811

Tetsuhito Muranaka

Wakkanai City Hospital

Masataka Yagisawa

Japanese Red Cross Kitami Hospital

Yasuyuki Kawamoto

Hokkaido University Hospital

Satoshi Yuki

Hokkaido University Graduate School of Medicine: Hokkaido Daigaku Daigakuin Igakuin

Atsushi Ishiguro

Teine Keijinkai Hospital: Teine Keijinkai Byoin

Masayoshi Dazai

Sapporo Medical Center NTT EC

Yoshimitsu Kobayashi

KKR Sapporo Medical Center: KKR Sapporo Iryo Center

\section{Susumu Sogabe}

KKR Sapporo Medical Center: KKR Sapporo Iryo Center

\section{Kazuaki Harada}

Hokkaido University Graduate School of Medicine: Hokkaido Daigaku Daigakuin Igakuin

\section{Kentaro Sawada}

Kushiro Rosai Hospital: Kushiro Rosai Byoin

\section{Takuto Miyagishima}

Kushiro Rosai Hospital: Kushiro Rosai Byoin

Yasushi Tsuji

Tonan Hospital

\section{Yasuka Kikuchi}

Hokkaido University Graduate School of Medicine: Hokkaido Daigaku Daigakuin Igakuin Yoshito Komatsu 


\section{Research Article}

Keywords: cancer treatment-induced bone loss, denosumab, gastrointestinal cancer, steroid-induced osteoporosis, glucocorticoid premedication

Posted Date: April 8th, 2021

DOI: https://doi.org/10.21203/rs.3.rs-400213/v1

License: (우 (i) This work is licensed under a Creative Commons Attribution 4.0 International License. Read Full License

Version of Record: A version of this preprint was published at Journal of Clinical Oncology on February 1st, 2017. See the published version at https://doi.org/10.1200/JC0.2017.35.4_suppl.TPS809. 


\section{Abstract \\ Purpose}

We previously reported that the periodic premedication of glucocorticoids during chemotherapy for gastrointestinal cancer (GIC) caused the reduction of bone mineral densities (BMD) (ESPRESSO-01). We conducted this study to evaluate the efficacy and safety of denosumab in the prevention of chemotherapyinduced decreased BMD.

\section{Methods}

Forty-two Japanese patients were studied. Denosumab was administered as a single $60 \mathrm{mg}$ subcutaneous injection before the start of chemotherapy. The primary endpoint was BMD change in the lumbar spine from baseline to 16 weeks. Secondary endpoints were changes in serum cross-linked N-telopeptides of type I collagen (sNTX) and bone alkaline phosphatase (sBAP), complications including hypocalcemia and osteonecrosis of the jaw, new bone fractures, changes in the Japanese Osteoporosis Quality of Life Questionnaire.

\section{Results}

Lumbar spine BMD significantly increased in $71.4 \%$ of cases: $2.772 \%(95 \% \mathrm{Cl}, 1.350-4.195 \%$ : $P<0.0001)$. There were also significant decreases in sNTX $(P=0.034)$ and sBAP levels $(P<0.001)$. Although one case (2.4\%) of jaw osteonecrosis was diagnosed, no fractures occurred. In a cross-trial comparison with ESPRESSO01, there was no significant difference in the incidence of National Cancer Institute Common Terminology Criteria for Adverse Events (CTCAE) grade 3 or higher adverse events. However, inclusion of grade $\leq 2$ showed significantly higher incidence of hypocalcemia with any CTCAE grade in ESPRESSO-02 (28.4\% in ESPRESSO$01,54.8 \%$ in ESPRESSO-02, $P=0.006)$

\section{Conclusion}

Denosumab administration prevented secondary BMD reduction in GIC patients undergoing chemotherapy, and was associated with decreased serum levels of the bone turnover markers BAP and NTX.

\section{Introduction:}

Bone health and the management of cancer treatment-induced bone loss (CTIBL) are important for cancer care because the diagnostic and therapeutic advances prolonged patients' survival. For example, in breast cancer the use of an aromatase inhibitor ( $\mathrm{Al})$ is associated with increased bone loss and incidence of fracture $[1,2]$ and, in prostate cancer, androgen deprivation therapy has been shown to correlate with a decrease in bone mineral density (BMD) and an increased fracture rate $[3,4]$. Also, in other cancer, secondary amenorrhoea and/or premature menopause induced by cytotoxic cancer treatments, rather than direct cytotoxic damage to bone cells, osteoporosis defined as CTIBL may occur and especially its endocrine effects lead to the bone loss 
observed in premenopausal women [5]. We previously reported that short-term periodic premedication of glucocorticoids (GCs) used with chemotherapy for gastrointestinal cancer (GIC) caused the reduction of BMD (ESPRESSO-01) [6]. In this study including not only postmenopausal women but also men or some premenopausal women, the average amount of BMD reduction rate was $1.89 \%$ from baseline and the decrease in BMD during 16-weeks GIC chemotherapy was comparable to what was seen after 12 months of adjuvant $\mathrm{Al}$ therapy.

Denosumab, a fully human monoclonal antibody against Receptor Activator of Nuclear Factor-Kappa B Ligand (RANKL), inhibits osteoclast activity and bone resorption and is being investigated for its clinical utility in men with castration-resistant prostate cancer and in postmenopausal women with breast cancer who are taking Als as an adjuvant endocrine therapy $[7,8]$, whereas there is no data for usefulness of denosumab in GIC patients. This prospective study was designed to investigate the efficacy and safety of denosumab in the prevention of CTIBL in GIC patients.

\section{Methods:}

\section{Study design}

This was a multicenter, prospective, interventional study designed to evaluate the efficacy and safety of denosumab in the prevention of CTIBL in Japanese GIC patients. The enrollment period was from April 2017 to February 2018. The protocol was performed in accordance with the Declaration of Helsinki, Japanese ethical guidelines on clinical research, and Ethical Guidelines for Clinical Studies and was registered with the University Hospital Medical Information Network (UMIN) Clinical Trials Registry (protocol ID UMIN000023855). Written informed consent was obtained from all patients, and protocol approval was obtained from the clinical research ethics review board of all participating institutions in Japan.

\section{Patients}

All patients fulfilled the following criteria: histologically confirmed GIC (colorectal and non-colorectal cancers), including esophageal, gastric, pancreatic, and biliary cancer; no prior treatment (e.g., radiation therapy or chemotherapy) for these cancers; scheduled premedication with periodic glucocorticoids to prevent chemotherapy-induced nausea and vomiting or allergic reactions that was scheduled weekly, biweekly, or triweekly and in which > 4-week steroid-free intervals were not allowed; age 40 to 90 years; a clinical risk score in the Japanese Society for Bone and Mineral Research (JSBMR) updated guidelines of 3 or more, which was defined as the optimal cutoff score for pharmacologic intervention to prevent steroid-induced osteoporosis [9]. The following exclusion criteria were used: previous or current regular steroid use, regular bisphosphonate or denosumab use; regimens that included steroid-free intervals of $>4$ weeks; patients in which dual-energy $x$-ray absorptiometry (DXA) could not be performed due to their inability to maintain posture; patients who had previously undergone a total or partial gastrectomy; premenopausal women; serum calcium levels under 8.0 $\mathrm{mg} / \mathrm{dl}$; serum creatinine levels $1.5 \mathrm{mg} / \mathrm{dl}$ or higher; ongoing dental treatment.

\section{BMD measurement}

BMD was measured with DXA of the lumbar spine ( $L 2, L 3$, and $L 4$ posteroanterior views) and the proximal left femur using the Hologic Discovery A or Ci or Horizon C (Hologic, Inc., Waltham, MA, USA) densitometers 
according to the manufacturer's protocols. A daily quality control test was performed using the manufacturerrecommended phantom to ensure that observed density changes were not due to machine and/or operator variability. All technicians responsible for measuring BMD were blinded. T-scores were calculated by dividing the difference between the patient's measured BMD and the mean BMD of healthy young adults matched for gender and ethnic group, and by expressing the difference relative to the young adult population standard deviation (SD). Z-scores were calculated by dividing the difference between the patient's measured BMD and the age-matched mean BMD expected for the patient's peers (a healthy normal subject matched for age, gender, and ethnic group) by the age-matched population SD.

\section{Bone turnover markers}

Two serum bone turnover markers (BTM), sBAP and sNTX, were measured. All assays were performed by SRL Inc. (Tokyo, Japan). All blood samples were collected in the morning after $\geq 8 \mathrm{~h}$ of fasting. sBAP levels were measured by a completely automated chemiluminescent enzyme immunoassay using a Beckman Coulter Access Ostase (Beckman Coulter, Inc., CA, USA) with a reference range of 3.7-20.9 $\mu \mathrm{g} / \mathrm{L}$ for men, 2.9-14.5 $\mu \mathrm{g} / \mathrm{L}$ for premenopausal women, and 3.8-22.6 $\mu \mathrm{g} / \mathrm{L}$ for postmenopausal women. SNTX levels were measured using a completely automated enzyme-linked immunosorbent assay (ELISA) with Osteomark NTx Serum ELISA Test Kits (Alere Inc., WA, USA). The reference ranges for men and premenopausal and postmenopausal women were 9.5-17.7, 7.5-16.5, and 10.7-24.0 nmol bone collagen equivalent (BCE)/L, respectively.

\section{Health-Related Quality of Life (QOL) Measurements}

We used the Japanese Osteoporosis Quality of Life Questionnaire (JOQOL), which was established by the JSBMR, to measure and assess the QOL of Japanese osteoporotic patients. The JOQOL instrument consists of 6 domains and 38 items that are based on the Osteoporosis Assessment Questionnaire and the Quality of Life Questionnaire of the European Foundation of Osteoporosis, with some added questions specific to Japanese lifestyle [10]. Higher scores indicated a better QOL, and each item was scored with a point scale from 0 to 4 , with a maximum total score of 152 points. The use of JOQOL was approved by the JSBMR secretariat.

\section{Diagnosis of Vertebral Fractures}

A vertebral fracture was defined as new when it occurred in a vertebra that was not fractured at baseline. A fracture adjudication process that consisted of quantitative morphometry (QM) by a central committee radiologist without any information on the patient was used to confirm the presence of a new vertebral fracture. QM was done as follows: an independent experienced research assistant marked six points per vertebra, defining the anterior (ha), posterior ( $\mathrm{hp})$, and middle ( $\mathrm{hm}$ ) vertebral heights [11]. In addition, the following ratios were calculated:

Anterior-posterior ratio $(\mathrm{APR})=\mathrm{ha} / \mathrm{hp}$

Middle-anterior ratio $(M A R)=\mathrm{hm} / \mathrm{ha}$

Middle-posterior ratio $($ MPR $)=\mathrm{hm} / \mathrm{hp}$

An existing fracture was defined as an APR of less than 0.75 or a MAR or MPR less than 0.8 at baseline. A new vertebral fracture was defined as an electronically measured decrease from baseline of at least $20 \%$ and a 
decrease of at least $4 \mathrm{~mm}$ in the each vertebral height.

\section{Outcome assessment}

The primary endpoint was BMD change in the L2-L4 lumbar spine (LS), which is considered the optimum site for monitoring treatment response [12], measured by DXA from baseline to 16 weeks after starting chemotherapy. Secondary endpoints included the percent changes from baseline in BTM levels, the safety profiles of denosumab, new fractures and changes in JOQOL and BMD in the femoral neck (FN) or total hip $(\mathrm{TH})$, which is considered the optimum sites for predicting the risk of a hip fracture $[13,14,15,16,17,18]$. For new fractures and jaw osteonecrosis we collected 2-year minimum follow-up data from the final case's denosumab start date to February 29th, 2020.

\section{Safety}

The safety of the chemotherapy regimen were assessed by the study physicians at each visit. The study physicians all assessed if any adverse events (AEs) were related to the chemotherapy drugs at that time. AEs were graded using the National Cancer Institute Common Terminology Criteria for Adverse Events (CTCAE), version 4.03.

\section{Statistical analysis}

In ESPRESSO-01 the average BMD reduction rate was - 1.89\%. Assuming that the lower limit of the $95 \%$ confidence of the BMD variation rate would exceed $-1.89 \%$, an estimated sample size of 36 was estimated based on a two-sided alpha value of 0.05 and a power of $90 \%$. We estimated that 45 patients would be needed to achieve the required number of final cases assuming $20 \%$ attrition. Patient characteristics were summarized using descriptive statistics. Steroid dose intensity was calculated by dividing the total dose by the number of treatment weeks. Changes in BMD, BTM levels, and JOQOL scores from baseline to 16 weeks after chemotherapy start were examined using a paired samples t-test. The cross-trial comparisons between ESPRESSO-01 and - 02 for adverse events were analyzed using the chi-squared test. Fisher's exact test was used when the frequency of any cell of the contingency table was $\leq 5$. Statistical significance was set at $P<$ 0.05. Statistical calculations were performed using SPSS for Macintosh (release 24.0; SPSS Inc., Chicago, IL, USA).

\section{Results:}

\section{Patients}

Of the forty-nine patients that were enrolled, two were not eligible because one died before study treatment and another refused to participate due to patient request. Five did not have a follow-up assessment because of the following reasons: one did not have a baseline BMD measurement; one was lost to follow up due to changing the hospital; one refused treatment continuation due to patient request; and 2 died before their week-16 assessment. The full analysis set for assessing primary outcome therefore included 42 patients (male, 18; female, 24; age, 50-81 years; median age, 68 years) (Fig. 1). Table 1 shows their baseline characteristics. All females were postmenopausal. 
Table 1

Baseline patient and treatment characteristics

\begin{tabular}{|c|c|}
\hline Characteristics & No. of patients (\%) \\
\hline Sex & $18(42.9)$ \\
\hline Male & $24(57.1)$ \\
\hline \multicolumn{2}{|l|}{ Female } \\
\hline Median age, years (range) & $68(50-81)$ \\
\hline ECOG PS & $32(76.2)$ \\
\hline 0 & $10(23.8)$ \\
\hline \multicolumn{2}{|l|}{1} \\
\hline Primary site & $6(14.3)$ \\
\hline Gastric cancer & $24(57.1)$ \\
\hline Colorectal cancer & $7(26.7)$ \\
\hline Pancreas cancer & $5(11.9)$ \\
\hline \multicolumn{2}{|l|}{ Biliary cancer } \\
\hline Chemotherapy Regimen & $17(40.5)$ \\
\hline CapeOX \pm Bmab & $4(9.5)$ \\
\hline FOLFOX + Pmab & $2(4.8)$ \\
\hline FOLFOXIRI + Bmab & $2(4.8)$ \\
\hline FOLFIRINOX & $4(9.5)$ \\
\hline Gem + nabPTX & $6(14.3)$ \\
\hline Gem + CDDP & $4(9.5)$ \\
\hline SOX \pm Tmab & $1(2.4)$ \\
\hline $\mid \mathrm{RI}+\mathrm{CDDP}$ & $1(2.4)$ \\
\hline IRIS + Bmab & $1(2.4)$ \\
\hline \multicolumn{2}{|l|}{$\mathrm{DTX}+\mathrm{CDDP}+\mathrm{S}-1$} \\
\hline Treatment Setting & $14(33.3)$ \\
\hline Adjuvant & $28(66.7)$ \\
\hline Non-adjuvant & \\
\hline
\end{tabular}




\begin{tabular}{|c|c|}
\hline Characteristics & No. of patients (\%) \\
\hline Treatment Duration (\%) & $10(23.8)$ \\
\hline Weekly & $10(23.8)$ \\
\hline Biweekly & $22(52.4)$ \\
\hline \multicolumn{2}{|l|}{ Triweekly } \\
\hline Chemotherapy Administration Cycles & 6.3 / $6.0(2.0-12.0)$ \\
\hline \multicolumn{2}{|l|}{ Mean / Median (range), cycles } \\
\hline Administration Days of GCs during 16 weeks & 11.5 / $8.5(2.0-70.0)$ \\
\hline \multicolumn{2}{|l|}{ Mean / Median (range), days } \\
\hline Cumulative dose of GCs during 16 weeks & 87.2 / 69.3 (9.9-272.2) \\
\hline \multicolumn{2}{|l|}{ Mean / Median (range), mg } \\
\hline Dose of GCs per chemotherapy cycles & 14.2 / $9.9(1.7-34.0)$ \\
\hline \multicolumn{2}{|l|}{ Mean / Median (range), mg/cycle } \\
\hline Dose Intensity of GCs & 5.5 / $4.3(0.6-17.0)$ \\
\hline \multicolumn{2}{|l|}{ Mean / Median (range), mg/week } \\
\hline \multicolumn{2}{|c|}{$\begin{array}{l}\text { Abbreviations: Bmab, bevacizumab; CapeOX, capecitabine (Cape)/oxaliplatin (OX); CDDP, cisplatin; DTX, } \\
\text { docetaxel; ECOG PS, Eastern Cooperative Oncology Group performance status; FOLFIRINOX, 5-fluorouracil } \\
\text { (5FU)/leucovorin (LV)//rinotecan (IRI)/OX; FOLFOX, 5FU/LV/OX; FOLFOXIRI 5FU/LV/OX/IRI, GC, } \\
\text { glucocorticoid; Gem, gemcitabine; nabPTX, nanoparticle albumin-bound paclitaxel (PTX); Pmab, } \\
\text { panitumumab; SOX, S-1/OX; Tmab, trastuzumab. }\end{array}$} \\
\hline
\end{tabular}

\section{BMD changes from baseline to 16 weeks post-therapy start}

There were statistically significant increases in the mean BMD from baseline to 16 weeks in all lesions: LS $\left(0.924\right.$ vs. $\left.0.949 \mathrm{~g} / \mathrm{cm}^{2}, P<0.0001\right), \mathrm{TH}\left(0.754\right.$ vs. $\left.0.761 \mathrm{~g} / \mathrm{cm}^{2}, P=0.004\right)$, and FN $\left(0.636\right.$ vs. $0.645 \mathrm{~g} / \mathrm{cm}^{2}, P=$ 0.022 ) (Table 2). Mean BMD percent changes at 16 weeks were $2.772 \%[N=42: 95 \%$ confidence interval $(\mathrm{Cl})$, 1.350 to $4.195 \%$ ], $1.360 \%(N=41: 95 \% \mathrm{Cl}, 0.526$ to $2.194 \%)$, and $1.945 \%(N=41: 95 \% \mathrm{Cl}, 0.375$ to $3.516 \%)$ in the LS, TH, and FN, respectively (Table 2). Increased LS BMDs were observed in 30 patients $(71.4 \%), 27$ (65.9\%) in the TH, and 24 (58.5\%) in the FN (Fig. 2). Similarly, there were also statistically significant increases in the Tscore and Z-score from baseline to 16 weeks in all lesions: LS ( -0.891 vs. $-0.633, P=0.001)$ and $(0.495$ vs. $0.657, P<0.0001), \mathrm{TH}(-1.254$ vs. $-1.191, P=0.020)$ and $(0.334$ vs. $0.405, P=0.003)$, and FN $(-1.578$ vs. $-1.500, P=0.033)$ and $(0.005$ vs. $0.107, P=0.015)$, respectively (Supplementary Material 1$)$. 
Table 2

Variations in BMD and BTMs from baseline to 16 weeks after the start of chemotherapy between ESPRESSO-

01 and -02

\begin{tabular}{|c|c|c|c|c|c|c|c|c|}
\hline & \multicolumn{4}{|c|}{$\begin{array}{l}\text { Denosumab } \\
\text { (ESPRESSO-02) }\end{array}$} & \multicolumn{4}{|c|}{$\begin{array}{l}\text { Observation (No treatment) } \\
\text { (ESPRESSO-01) }\end{array}$} \\
\hline & $\begin{array}{l}\text { Baseline } \\
\text { Mean } \\
( \pm \text { SD) } \\
{[n]}\end{array}$ & $\begin{array}{l}16 \\
\text { weeks } \\
\text { Mean } \\
( \pm \text { SD) } \\
{[n]}\end{array}$ & $\begin{array}{l}\text { Percent } \\
\text { changes } \\
\text { Mean \% } \\
( \pm \text { SD) } \\
{[\text { ] }}\end{array}$ & $P^{*}$ & $\begin{array}{l}\text { Baseline } \\
\text { Mean } \\
( \pm \text { SD) } \\
\text { [n] }\end{array}$ & $\begin{array}{l}16 \\
\text { weeks } \\
\text { Mean } \\
( \pm \text { SD) } \\
{[n]}\end{array}$ & $\begin{array}{l}\text { Percent } \\
\text { changes } \\
\text { Mean \% } \\
( \pm \text { SD) } \\
\text { [n] }\end{array}$ & $P^{*}$ \\
\hline \multicolumn{9}{|l|}{$\begin{array}{l}\text { BMD } \\
\left(\mathrm{g} / \mathrm{cm}^{2}\right)\end{array}$} \\
\hline \multirow[t]{2}{*}{$\begin{array}{l}\text { Lumbar } \\
\text { spine }\end{array}$} & $\begin{array}{l}0.924 \\
( \pm \\
0.178)\end{array}$ & $\begin{array}{l}0.949 \\
( \pm \\
0.184)\end{array}$ & $\begin{array}{l}+2.772 \\
( \pm \\
4.565)\end{array}$ & $\begin{array}{l}< \\
0.0001\end{array}$ & $\begin{array}{l}1.061 \\
( \pm \\
0.223)\end{array}$ & $\begin{array}{l}1.041 \\
( \pm \\
0.221)\end{array}$ & $\begin{array}{l}-1.890 \\
( \pm \\
3.358)\end{array}$ & $\begin{array}{l}< \\
0.0001\end{array}$ \\
\hline & {$[\mathrm{n}=42]$} & $\begin{array}{l}{[n=} \\
42]\end{array}$ & {$[\mathrm{n}=42]$} & & {$[n=74]$} & {$[n=74]$} & {$[n=74]$} & \\
\hline \multirow[t]{2}{*}{ Total hip } & $\begin{array}{l}0.754 \\
( \pm \\
0.145)\end{array}$ & $\begin{array}{l}0.761 \\
( \pm \\
0.138)\end{array}$ & $\begin{array}{l}+1.360 \\
( \pm \\
2.643)\end{array}$ & 0.004 & $\begin{array}{l}0.886 \\
( \pm \\
0.160)\end{array}$ & $\begin{array}{l}0.864 \\
( \pm \\
0.165)\end{array}$ & $\begin{array}{l}-2.243 \\
( \pm \\
5.786)\end{array}$ & 0.002 \\
\hline & {$[n=41]$} & $\begin{array}{l}{[n=} \\
42]\end{array}$ & {$[n=41]$} & & {$[n=74]$} & {$[n=73]$} & {$[n=73]$} & \\
\hline \multirow[t]{2}{*}{$\begin{array}{l}\text { Femoral } \\
\text { neck }\end{array}$} & $\begin{array}{l}0.636 \\
( \pm \\
0.120)\end{array}$ & $\begin{array}{l}0.645 \\
( \pm \\
0.112)\end{array}$ & $\begin{array}{l}+1.945 \\
( \pm \\
4.975)\end{array}$ & 0.022 & $\begin{array}{l}0.742 \\
( \pm \\
0.154)\end{array}$ & $\begin{array}{l}0.723 \\
( \pm \\
0.148)\end{array}$ & $\begin{array}{l}-2.048 \\
( \pm \\
4.559)\end{array}$ & $\begin{array}{l}< \\
0.0001\end{array}$ \\
\hline & {$[n=41]$} & $\begin{array}{l}{[n=} \\
42]\end{array}$ & {$[n=41]$} & & {$[n=74]$} & {$[n=73]$} & {$[n=73]$} & \\
\hline \multirow[t]{2}{*}{$\operatorname{sBAP}(\mu \mathrm{g} / \mathrm{L})$} & $\begin{array}{l}15.529 \\
( \pm \\
8.647)\end{array}$ & $\begin{array}{l}9.819 \\
( \pm \\
3.080)\end{array}$ & $\begin{array}{l}-29.441 \\
( \pm \\
22.348)\end{array}$ & $\begin{array}{l}< \\
0.0001\end{array}$ & $\begin{array}{l}14.900 \\
( \pm \\
7.787)\end{array}$ & $\begin{array}{l}18.056 \\
( \pm \\
10.309)\end{array}$ & $\begin{array}{l}+ \\
26.351 \\
( \pm\end{array}$ & 0.010 \\
\hline & {$[\mathrm{n}=42]$} & $\begin{array}{l}{[n=} \\
42]\end{array}$ & {$[n=42]$} & & {$[n=72]$} & {$[n=73]$} & {$[n=71]$} & \\
\hline \multirow[t]{2}{*}{$\begin{array}{l}\text { sNTX } \\
\text { (nmolBCE/L) }\end{array}$} & $\begin{array}{l}23.637 \\
( \pm \\
31.773)\end{array}$ & $\begin{array}{l}10.777 \\
( \pm \\
4.403)\end{array}$ & $\begin{array}{l}-37.948 \\
( \pm \\
29.498)\end{array}$ & 0.034 & $\begin{array}{l}19.124 \\
( \pm \\
5.172)\end{array}$ & $\begin{array}{l}20.319 \\
( \pm \\
8.051)\end{array}$ & $\begin{array}{l}+9.505 \\
( \pm \\
43.081)\end{array}$ & 0.136 \\
\hline & {$[n=30]$} & $\begin{array}{l}{[\mathrm{n}=} \\
30]\end{array}$ & {$[n=30]$} & & {$[n=72]$} & {$[n=73]$} & {$[n=71]$} & \\
\hline
\end{tabular}

Changes in BTMs 
There was a significant decrease in sNTX levels from baseline to 16 weeks: -37.9 nmolBCE/L (95\% Cl, -49.0 to $-27.0 \mathrm{nmolBCE} / \mathrm{L})(P=0.034)$. There was also a significant decrease in sBAP levels: $-29.4 \mu \mathrm{g} / \mathrm{L}(95 \% \mathrm{Cl},-$ 36.4 to $-22.5 \mu \mathrm{g} / \mathrm{L})(P<0.0001)$ (Table 2$)$.

\section{Safety}

Although there was no difference in serum albumin levels from baseline to 16 weeks, significant decreases not only in serum calcium levels $(P<0.0001)$ but also total serum calcium levels corrected for albumin $(P<0.0001)$ were observed (Supplementary Material 1). In a cross-trial comparison between ESPRESSO-01 and - 02 , although there was no significant difference in CTCEA grade 3 or higher AE, grade 2 AEs and below were added the incidence of hypocalcemia with any CTCAE grade was significantly higher in ESPRESSO-02 $(28.4 \%$ in ESPRESSO-01, 54.8\% in ESPRESSO-02, $P=0.006)$ (Table 3$)$. Although clinical fractures occurred in $0 / 42(0 \%)$ and $4 / 74$ (5.4\%) subjects who received denosumab and no treatment, respectively, there was no statistical difference between ESPRESSO-01 and - 02. In the 2-year minimum follow-up from ESPRESSO-02 (median observation periods: 25 months, range 5-34 months), 29 patients (69\%) discontinued denosumab but no new fractures with or without denosumab discontinuation (Table 4). Although there was no statistical difference between ESPRESSO-01 and - 02, one subject (2.4\%) in ESPRESSO-02 was diagnosed with jaw osteonecrosis. No severe hematologic adverse events or laboratory test abnormalities with CTCAE grade 3 or higher AE were more common in ESPRESSO-02 than ESPRESSO-01. 
Table 3

Summary of adverse events between ESPRESSO-01 and - 02

\begin{tabular}{|c|c|c|c|c|c|c|}
\hline \multirow{3}{*}{ Events } & \multicolumn{2}{|c|}{ ESPRESSO-02 $(n=42)$} & \multicolumn{2}{|c|}{ ESPRESSO-01 $(n=74)$} & \multicolumn{2}{|l|}{$P$} \\
\hline & $\begin{array}{l}\text { Any } \\
\text { Grade* }\end{array}$ & $\begin{array}{l}\text { Grade } 3 \text { or } \\
\text { higher }\end{array}$ & $\begin{array}{l}\text { Any } \\
\text { Grade* }\end{array}$ & $\begin{array}{l}\text { Grade } 3 \text { or } \\
\text { higher }\end{array}$ & $\begin{array}{l}\text { Any } \\
\text { Grade }\end{array}$ & $\begin{array}{l}\text { Grade } 3 \text { or } \\
\text { higher }\end{array}$ \\
\hline & $\mathrm{n}(\%)$ & $\mathrm{n}(\%)$ & $\mathrm{n}(\%)$ & $\mathrm{n}(\%)$ & & \\
\hline $\begin{array}{l}\text { Bone related AE } \\
\text { (Overall) }\end{array}$ & $\begin{array}{l}24 \\
(57.1)\end{array}$ & $2(4.8)$ & $\begin{array}{l}24 \\
(32.4)\end{array}$ & $4(5.4)$ & 0.011 & $1^{\dagger}$ \\
\hline Osteonecrosis of jaw & $1(2.4)$ & $1(2.4)$ & $0(0)$ & $0(0)$ & $0.362^{\dagger}$ & $0.362^{\dagger}$ \\
\hline Clinical Fracture & $0(0)$ & $0(0)$ & $4(5.4)$ & $4(5.4)$ & $0.295^{\dagger}$ & $0.295^{\dagger}$ \\
\hline Hypocalcemia & $\begin{array}{l}23 \\
(54.8)\end{array}$ & $1(2.4)$ & $\begin{array}{l}21 \\
(28.4)\end{array}$ & $0(0)$ & 0.006 & $0.362^{\dagger}$ \\
\hline $\begin{array}{l}\text { Hematologic AE } \\
\text { (Overall) }\end{array}$ & $\begin{array}{l}40 \\
(95.2)\end{array}$ & $17(40.5)$ & $\begin{array}{l}71 \\
(95.9)\end{array}$ & $28(37.8)$ & 1 & 0.844 \\
\hline Anemia & \multirow[t]{2}{*}{$\begin{array}{l}32 \\
(76.2)\end{array}$} & $3(7.1)$ & \multirow[t]{2}{*}{$\begin{array}{l}61 \\
(82.4)\end{array}$} & $9(12.2)$ & 0.471 & $0.532^{\dagger}$ \\
\hline Leukopenia & & $6(14.3)$ & & $8(10.8)$ & 0.113 & 0.768 \\
\hline Neutropenia & $\begin{array}{l}28 \\
(66.7)\end{array}$ & $16(38.1)$ & $(81.1)$ & $16(21.6)$ & 0.325 & 0.083 \\
\hline \multirow[t]{2}{*}{ Thrombocytopenia } & $\begin{array}{l}29 \\
(69.0)\end{array}$ & $3(7.1)$ & $\begin{array}{l}44 \\
(59.5)\end{array}$ & $5(6.8)$ & 1 & $1^{\dagger}$ \\
\hline & $\begin{array}{l}26 \\
(61.9)\end{array}$ & & $\begin{array}{l}47 \\
(63.5)\end{array}$ & & & \\
\hline $\begin{array}{l}\text { Laboratory test } \\
\text { abnormalities }\end{array}$ & $\begin{array}{l}42 \\
(100)\end{array}$ & $7(16.7)$ & 72(97.3) & $20(27.0)$ & 0.534 & 0.256 \\
\hline (Overall) & & & & & & \\
\hline
\end{tabular}




\begin{tabular}{|c|c|c|c|c|c|c|}
\hline \multirow[b]{2}{*}{ AST elevation } & \multicolumn{2}{|c|}{ ESPRESSO-02 $(n=42)$} & \multicolumn{2}{|c|}{ ESPRESSO-01 $(n=74)$} & \multicolumn{2}{|l|}{$P$} \\
\hline & \multirow[t]{2}{*}{$\begin{array}{l}27 \\
(64.3)\end{array}$} & \multirow{2}{*}{$\begin{array}{l}2(4.8) \\
2(4.8)\end{array}$} & $\begin{array}{l}36 \\
(48.6)\end{array}$ & \multirow{2}{*}{$\begin{array}{l}3(4.1) \\
2(2.7)\end{array}$} & 0.123 & $1^{\dagger}$ \\
\hline ALT elevation & & & \multirow{2}{*}{$\begin{array}{l}31 \\
(41.9)\end{array}$} & & \multirow{2}{*}{$\begin{array}{l}0.334 \\
0.694\end{array}$} & $0.620^{\dagger}$ \\
\hline GGT elevation & $\begin{array}{l}22 \\
(52.4)\end{array}$ & $5(11.9)$ & & $9(12.2)$ & & \\
\hline Bilirubin elevation & $\begin{array}{l}17 \\
(40.5)\end{array}$ & & $\begin{array}{l}27 \\
(36.5)\end{array}$ & $6(8.1)$ & $<.001$ & $0.419^{\dagger}$ \\
\hline $\begin{array}{l}\text { Hypoalbuminemia } \\
\text { Hypernatremia }\end{array}$ & $\begin{array}{l}7 \\
(16.7)\end{array}$ & $0(0)$ & $\begin{array}{l}39 \\
(52.7)\end{array}$ & $3(4.1)$ & 0.419 & $0.552^{\dagger}$ \\
\hline \multirow{2}{*}{$\begin{array}{l}\text { Hyponatremia } \\
\text { Hyperkalemia }\end{array}$} & \multirow{2}{*}{$\begin{array}{l}41 \\
(97.6)\end{array}$} & $0(0)$ & 68 & $0(0)$ & \multirow{2}{*}{$\begin{array}{l}0.567^{\dagger} \\
0.003\end{array}$} & $1^{\dagger}$ \\
\hline & & $0(0)$ & (91.9) & $0(0)$ & & $1^{\dagger}$ \\
\hline Hypokalemia & $4(9.5)$ & $1(2.4)$ & (14.9) & $1(1.4)$ & \multirow{2}{*}{$\begin{array}{l}0.135 \\
0.092\end{array}$} & $1^{\dagger}$ \\
\hline Creatinine elevation & $\begin{array}{l}19 \\
(45.2)\end{array}$ & $0(0)$ & $\begin{array}{l}14 \\
(18.9)\end{array}$ & $0(0)$ & & $1^{\dagger}$ \\
\hline Hematuria & \multirow{2}{*}{$\begin{array}{l}8 \\
(19.0)\end{array}$} & $0(0)$ & $6(8.1)$ & $3(4.1)$ & \multirow{3}{*}{$\begin{array}{l}1 \\
0.540\end{array}$} & \\
\hline Proteinuria & \multirow{2}{*}{\multicolumn{2}{|c|}{$\begin{array}{l}17 \\
(40.5)\end{array}$}} & & $3(4.1)$ & & \\
\hline & & & $\begin{array}{l}18 \\
(24.3)\end{array}$ & & & $0.552^{\dagger}$ \\
\hline & \multicolumn{2}{|l|}{$\begin{array}{l}5 \\
(11.9)\end{array}$} & \multicolumn{2}{|l|}{$\begin{array}{l}19 \\
(25.7)\end{array}$} & & $0.552^{\dagger}$ \\
\hline & \multicolumn{2}{|l|}{$\begin{array}{l}7 \\
(16.7)\end{array}$} & \multicolumn{2}{|l|}{$\begin{array}{l}11 \\
(14.9)\end{array}$} & & \\
\hline & \multicolumn{2}{|l|}{$\begin{array}{l}12 \\
(28.6)\end{array}$} & \multicolumn{2}{|l|}{$\begin{array}{l}26 \\
(35.1)\end{array}$} & & \\
\hline $\begin{array}{l}\text { Non-hematologic AE } \\
\text { (Overall) }\end{array}$ & $\begin{array}{l}38 \\
(90.5)\end{array}$ & $7(16.7)$ & $\begin{array}{l}61 \\
(82.4)\end{array}$ & $7(9.5)$ & $0.285^{\dagger}$ & 0.374 \\
\hline
\end{tabular}




\begin{tabular}{|c|c|c|c|c|c|c|}
\hline & \multicolumn{2}{|c|}{ ESPRESSO-02 $(n=42)$} & \multicolumn{2}{|c|}{ ESPRESSO-01 $(n=74)$} & \multicolumn{2}{|l|}{$P$} \\
\hline & 7 & $0(0)$ & 24 & $0(0)$ & 0.082 & $1^{\dagger}$ \\
\hline Diarrhea & \multirow[b]{2}{*}{$\begin{array}{l}10 \\
(23.8)\end{array}$} & $2(4.8)$ & \multirow[b]{2}{*}{$\begin{array}{l}23 \\
(31.1)\end{array}$} & $1(1.4)$ & 0.521 & $0.297^{\dagger}$ \\
\hline Nausea & & $0(0)$ & & $1(1.4)$ & 0.841 & \\
\hline Vomiting & \multirow[t]{2}{*}{$\begin{array}{l}16 \\
(38.1)\end{array}$} & $0(0)$ & $\begin{array}{l}26 \\
(35.1)\end{array}$ & $0(0)$ & $0.323^{\dagger}$ & $1^{\dagger}$ \\
\hline Stomatitis & & $0(0)$ & \multirow{2}{*}{$9(12.2)$} & $0(0)$ & 1 & \\
\hline Fatigue & $2(4.8)$ & $0(0)$ & & $0(0)$ & 1 & $1^{\dagger}$ \\
\hline Anorexia & $\begin{array}{l}6 \\
(14.3)\end{array}$ & $3(7.1)$ & $\begin{array}{l}11 \\
(14.9)\end{array}$ & $4(5.4)$ & 1 & $1^{\dagger}$ \\
\hline Dysgeusia & $\begin{array}{l}16 \\
(38.1)\end{array}$ & $0(0)$ & $\begin{array}{l}28 \\
(37.8)\end{array}$ & $0(0)$ & $0.217^{\dagger}$ & 0.703 \\
\hline Hiccups & \multicolumn{2}{|l|}{$\begin{array}{l}19 \\
(45.2)\end{array}$} & \multicolumn{2}{|l|}{$\begin{array}{l}34 \\
(45.9)\end{array}$} & $0.485^{\dagger}$ & $1^{\dagger}$ \\
\hline Hoarseness & $\begin{array}{l}5 \\
(11.9)\end{array}$ & $0(0)$ & $\begin{array}{l}17 \\
(23.0)\end{array}$ & $0(0)$ & $0.419^{\dagger}$ & $1^{\dagger}$ \\
\hline Epistaxis & $2(4.8)$ & $0(0)$ & $7(9.5)$ & $0(0)$ & $0 \cap 00^{\dagger}$ & $1^{\dagger}$ \\
\hline Hyperpigmentation & $3(7.1)$ & $0(0)$ & $5(6.8)$ & $0(0)$ & \multirow{2}{*}{0.441} & \multirow{2}{*}{$1^{\dagger}$} \\
\hline \multirow{2}{*}{$\begin{array}{l}\text { Peripheral } \\
\text { neuropathy }\end{array}$} & $1(2.4)$ & $0(0)$ & $6(8.1)$ & $1(1.4)$ & & \\
\hline & $2(4.8)$ & $0(0)$ & 18 & $0(0)$ & $1^{\dagger}$ & $1^{\top}$ \\
\hline (2) & 24 & $0(0)$ & & $0(0)$ & $1^{\dagger}$ & $1^{\dagger}$ \\
\hline Hand-Foot skin & $(57.1)$ & $0(0)$ & $\begin{array}{l}36 \\
(48.6)\end{array}$ & $0(0)$ & 0.819 & $1^{\dagger}$ \\
\hline reaction & $4(9.5)$ & $0(0)$ & $7(9.5)$ & $0(0)$ & $1^{\dagger}$ & $1^{\dagger}$ \\
\hline Paronychia & $4(9.5)$ & $1(2.4)$ & $6(8.1)$ & $0(0)$ & $0.652^{\dagger}$ & $1^{\dagger}$ \\
\hline Arthritis & $\begin{array}{l}10 \\
(23.8)\end{array}$ & $1(2.4)$ & 16 & $0(0)$ & $0.297^{\dagger}$ & $1^{\dagger}$ \\
\hline Pneumonitis & $2(4.8)$ & $0(0)$ & & $2(2.7)$ & & \\
\hline Allergic reaction & $1(2.4)$ & $0(0)$ & $5(6.8)$ & $0(0)$ & 11 & $0.362^{\top}$ \\
\hline Edema limbs & $2(4.8)$ & $0(0)$ & $4(5.4)$ & $0(0)$ & $0.255^{\dagger}$ & $0.362^{\dagger}$ \\
\hline Watering eyes & $1(2.4)$ & & $1(1.4)$ & & $1^{\dagger}$ & 0.534 \\
\hline & $1(2.4)$ & & $3(4.1)$ & & & $1^{\dagger}$ \\
\hline & $1(2.4)$ & & $7(9.5)$ & & & $1^{\dagger}$ \\
\hline & & & $1(2.4)$ & & & \\
\hline
\end{tabular}




\section{ESPRESSO-02 $(n=42)$}

ESPRESSO-01 $(n=74)$

$P$

$P$ value data are results of the chi-square test between ESPRESSO-01 and - 02 .

*Grading are evaluated based on Common Terminology Criteria for Adverse Events v4.03

${ }^{\dagger}$ Fisher's exact test.

Abbreviations: AE, adverse events; AST, aspartate aminotransferase; ALT, alanine aminotransferase; GGT, gamma glutamyl transferase. 
Table 4

2-year follow-up for bone fractures and jaw osteonecrosis

\begin{tabular}{|c|c|c|c|c|}
\hline & $\begin{array}{l}\text { Patients }(n=42) \\
\text { no./total no. (\%) }\end{array}$ & $\begin{array}{l}\text { Median observation } \\
\text { period*, months (range) }\end{array}$ & $\begin{array}{l}\text { New bone } \\
\text { fracture }\end{array}$ & $\begin{array}{l}\text { Jaw } \\
\text { osteonecrosis }\end{array}$ \\
\hline $\begin{array}{l}\text { Denosumab } \\
\text { continuation }^{a}, \mathrm{n}(\%)\end{array}$ & $n=42$ & $25.0(5-34)$ & & \\
\hline Continue & $13 / 42(31)$ & $24.0(5-34)$ & $0 / 42(0)$ & $0 / 42(0)$ \\
\hline Discontinue & 29/42 (69) & $25.0(5-32)$ & $0 / 42(0)$ & $1 / 42(2)$ \\
\hline $\begin{array}{l}\text { End of } \\
\text { chemotherapy }\end{array}$ & $15 / 42(36)$ & $27.0(21-32)$ & $0 / 42(0)$ & $0 / 42(0)$ \\
\hline $\begin{array}{l}\text { Poor general } \\
\text { condition }\end{array}$ & $3 / 42$ (7) & $7.0(5-23)$ & $0 / 42(0)$ & $0 / 42(0)$ \\
\hline $\begin{array}{l}\text { Adverse } \\
\text { events }\end{array}$ & $1 / 42(2)$ & 26.0 & $0 / 42(0)$ & $1 / 42(2)$ \\
\hline $\begin{array}{l}\text { Investigator's } \\
\text { decision }\end{array}$ & 10/42 (24) & $20.5(9-29)$ & $0 / 42(0)$ & $0 / 42(0)$ \\
\hline Adjuvant setting, $\mathrm{n}(\%)$ & $n=14$ & $26.5(21-32)$ & & \\
\hline Continue & $2 / 14(14)^{b}$ & $26.0(25-27)$ & $0 / 14(0)^{b}$ & $0 / 14(0)^{b}$ \\
\hline Discontinue & $12 / 14(86)^{b}$ & $26.5(21-32)$ & $0 / 14(0)^{b}$ & $0 / 14(0)^{b}$ \\
\hline $\begin{array}{l}\text { End of } \\
\text { chemotherapy }\end{array}$ & $12 / 14(86)^{b}$ & $26.5(21-32)$ & $0 / 14(0)^{b}$ & $0 / 14(0)^{b}$ \\
\hline $\begin{array}{l}\text { Poor general } \\
\text { condition }\end{array}$ & $0 / 14(0)^{b}$ & - & $0 / 14(0)^{b}$ & $0 / 14(0)^{b}$ \\
\hline $\begin{array}{l}\text { Adverse } \\
\text { events }\end{array}$ & $0 / 14(0)^{b}$ & - & $0 / 14(0)^{b}$ & $0 / 14(0)^{b}$ \\
\hline $\begin{array}{l}\text { Investigator's } \\
\text { decision }\end{array}$ & $0 / 14(0)^{b}$ & - & $0 / 14(0)^{b}$ & $0 / 14(0)^{b}$ \\
\hline $\begin{array}{l}\text { Non-adjuvant setting, } \\
\mathrm{n}(\%)\end{array}$ & $\mathrm{n}=28$ & $23.5(5-34)$ & & \\
\hline Continue & $11 / 28(39)^{c}$ & $15.0(5-34)$ & $0 / 28(0)^{c}$ & $0 / 28(0)^{c}$ \\
\hline Discontinue & $17 / 28(61)^{c}$ & $24.0(5-31)$ & $0 / 28(0)^{c}$ & $0 / 28(0)^{c}$ \\
\hline $\begin{array}{l}\text { End of } \\
\text { chemotherapy }\end{array}$ & $3 / 28(11)^{\mathrm{C}}$ & $30.0(29-31)$ & $0 / 28(0)^{c}$ & $0 / 28(0)^{c}$ \\
\hline $\begin{array}{l}\text { Poor general } \\
\text { condition }\end{array}$ & $3 / 28(11)^{\mathrm{C}}$ & $7.0(5-23)$ & $0 / 28(0)^{c}$ & $0 / 28(0)^{c}$ \\
\hline $\begin{array}{l}\text { Adverse } \\
\text { events }\end{array}$ & $1 / 28(4)^{c}$ & 26.0 & $0 / 28(0)^{c}$ & $1 / 28(4)^{c}$ \\
\hline
\end{tabular}




\begin{tabular}{|c|c|c|c|c|}
\hline & $\begin{array}{l}\text { Patients }(n=42) \\
\text { no./total no. }(\%)\end{array}$ & $\begin{array}{l}\text { Median observation } \\
\text { period*, months (range) }\end{array}$ & $\begin{array}{l}\text { New bone } \\
\text { fracture }\end{array}$ & $\begin{array}{l}\text { Jaw } \\
\text { osteonecrosis }\end{array}$ \\
\hline $\begin{array}{l}\text { Investigator's } \\
\text { decision }\end{array}$ & $10 / 28(36)^{c}$ & $20.5(9-29)$ & $0 / 28(0)^{c}$ & $0 / 28(0)^{c}$ \\
\hline \multicolumn{5}{|c|}{ a Data cut-off date: 29 February 2020.} \\
\hline \multicolumn{5}{|c|}{ b Percent calculated based on number of adjuvant setting cases. } \\
\hline \multicolumn{5}{|c|}{ c Percent calculated based on number of non-adjuvant setting cases. } \\
\hline \multicolumn{5}{|c|}{${ }^{*}$ Refers to the time from initiating denosumab treatment until data cut-off date or patient death. } \\
\hline
\end{tabular}

\section{QOL Scores}

Since QOL was not measured by ESPRESSO-01, it was not possible to compare differences between the two trials. No domains were exacerbated by denosumab (Supplementary Material 2).

\section{Discussion:}

This work observed that prophylactic denosumab can suppress CTIBL observed during GIC chemotherapy, which met our primary endpoint. BMD increase can be regarded as a surrogate for drug-mediated fracture prevention in CTIBL [19], and no pathologic fractures occurred. JSBMR guidelines identify the following as risk factors for a pathologic fracture in patients considering oral GC therapy for 3 months or longer: an age of 65 years or older, prednisolone $\geq 7.5 \mathrm{mg}$ /day (or its equivalent), a prior fragility fracture, and lumbar $\mathrm{BMD}<70 \%$ of the YAM are all assigned a score $\geq 3$ as single risk factors, and both alendronate and risedronate are recommended for high risk patients as first-line treatment [9]. Denosumab has been found to be more effective at increasing BMD than alendronate [20] or risedronate [21], and can be a first choice for the treatment of CTIBL in patients whose JSBMR clinical risk scores were 3 or more. As oral intake is difficult in some patients with GIC, the subcutaneous route of denosomab and its infrequent dosing once every 6 months may also be preferable from a compliance perspective.

Regarding the safety of denosumab, one case of jaw osteonecrosis was observed, although it was not serious. This occurred despite the fact that the protocol stipulated that all participants should undergo dental checks before starting treatment and continue to take prescribed calcium carbonate/cholecalciferol (vitamin D3)/magnesium carbonate combination tablets during the study period. Although cross-trial comparisons with ESPRESSO-01 showed no significant difference in the frequency of jaw osteonecrosis or severe hypocalcemia with CTCAE grade 3 or higher AE (Table 3), in the analysis including CTCAE grade 2 or lower AEs the incidence of hypocalcemia in the ESPRESSO-02 group was higher than in ESPRESSO-01 without denosumab treatment. Therefore, serum calcium level monitoring and oral assessment are important when using denosumab.

\section{Study limitations}

This study had notable limitations. First, its sample size was small. Since the number of cases was set based on the statistical hypothesis regarding BMD changes in this study, it is possible that this number was insufficient for evaluating the risk of fracture. Second, the observation period was short. It is well-known that 
denosumab discontinuation is associated with multiple spontaneous vertebral fractures. As described in several case series and in the post-hoc follow-up of the FREEDOM trial, patients presented with a median of five (1-11) vertebral fractures 7-20 months (median 11) after their last denosumab injection $[22,23,24,25$, $26,27,28]$. In our study the protocol did not specify whether denosumab would be continued after the 16-week intervention period, so we are not able to accurately assess the risk of denosumab discontinuation. In a 2-year follow-up post-hoc analysis from the start date of denosumab to the data cut-off date (29 February 2020), although no fractures were observed with or without denosumab discontinuation, the number of cases is too small to assess the incidence of fractures that may increase with denosumab discontinuation. This was an important limitation of this study and it should be noted that the sudden discontinuation of denosumab may increase the risk of multiple fractures. Further studies are needed to evaluate how the risk of fracture changes over the duration of denosumab therapy and after its discontinuation. Third, since this study is a single-arm intervention study, it cannot be directly compared with a non-intervention group. As the exploratory ESPRESSO01 trial revealed a high degree of BMD loss in patients who received GIC chemotherapy, it was ethically difficult to conduct a comparative study with an untreated group. This comparison is therefore only possible if a historical control was used. In addition, since we have not compared denosumab with other drugs such as alendronate, risedronate, and zoledronate, we do not know the superiority of denosumab against other drugs in this setting. Interestingly, Lin et al [29] found that after 1 year of treatment denosumab was more effective at increasing bone mass than alendronate. However, the fracture risk reduction was the same with both medications. In a mixed treatment comparison meta-analysis, Migliore et al [30] reported that compared with a placebo, zoledronate had the highest probability $(52 \%)$ of being the most effective treatment in the prevention of vertebral fractures, followed by denosumab ( $46 \%$ probability) and ibandronate, alendronate, and risedronate. Further verification is needed to determine whether denosumab is superior to other agents in terms of its ability to reduce the risk of fractures due to CTIBL.

\section{Conclusions:}

To our knowledge, this is the first study to evaluate the efficacy and safety of denosumab in GI cancer patients treated with chemotherapy. We showed that prophylactic administration of denosumab increased BMD levels and decreased sBAP and sNTX levels decreased during 16 weeks of chemotherapy. It should be noted that there is a risk of osteonecrosis of the jaw and hypocalcemia, but denosumab is considered to be one of the important preventive treatment options for CTIBL in GI chemotherapy. Further studies are needed on the longterm effects, especially the risk of fractures if discontinued, and whether there are benefits in terms of life prognosis, quality of life and cost.

\section{Declarations}

\section{Funding: N/A}

Conflicts of interest/Competing interests: Michio Nakamura has received honoraria from Daiichi Sankyo. Yoshito Komatsu has received honoraria and institutional research funding from Daiichi Sankyo. Tetsuhito Muranaka, Masataka Yagisawa, Yasuyuki Kawamoto, Satoshi Yuki, Atsushi Ishiguro, Masayoshi Dazai, Yoshimitsu Kobayashi, Susumu Sogabe, Kazuaki Harada, Kentaro Sawada, Takuto Miyagishima, Yasushi Tsuji, and Yasuka Kikuchi declare that they have no conflict of interest. 
Availability of data and material: The data that support the findings of this study are available from the corresponding author, Michio Nakamura, upon reasonable request.

\section{Code availability: N/A}

Author's contributions: Conception and design of study: Michio Nakamura and Yoshito Komatsu; provision of study materials or patients: all authors; collection and assembly of data: all authors; data analysis and interpretation: all authors; manuscript writing: all authors; final approval of manuscript: all authors.

Ethics approval: This study was performed in accordance with the Declaration of Helsinki, Japanese ethical guidelines on clinical research, and Ethical Guidelines for Clinical Studies and approved by each clinical research ethics review boards of all participating institutions.

Consent to participate: Written informed consent was obtained from all patients before participating in this study.

Consent for publication: Written informed consent was obtained from all patients before participating in this study.

\section{References}

1. Coleman RE, Banks LM, Girgis SI et al (2007) Skeletal effects of exemestane on bone-mineral density, bone biomarkers, and fracture incidence in postmenopausal women with early breast cancer participating in the Intergroup Exemestane Study (IES): a randomised controlled study. Lancet Oncol 8:119-127

2. Eastell R, Adams JE, Coleman RE et al (2008) Effect of anastrozole on bone mineral density: 5-year results from the anastrozole, tamoxifen, alone or in combination trial 18233230. J Clin Oncol 26:1051-1057

3. Shahinian VB, Kuo YF, Freeman JL, Goodwin JS (2005) Risk of fracture after androgen deprivation for prostate cancer. N Engl J Med 352:154-164

4. Smith MR, Lee WC, Brandman J, Wang Q, Botteman M, Pashos CL (2005) Gonadotropin-releasing hormone agonists and fracture risk: a claims-based cohort study of men with nonmetastatic prostate cancer. J Clin Oncol 23:7897-7903

5. Coleman RE, Rathbone E, Brown JE (2013) Management of cancer treatment-induced bone loss. Nat Rev Rheumatol 9:365-374

6. Nakamura M, Ishiguro A, Muranaka T et al (2017) A prospective observational study on effect of shortterm periodic steroid premedication on bone metabolism in gastrointestinal cancer (ESPRESSO-01). Oncologist 22:592-600

7. Gnant M, Pfeiler G, Dubsky PC et al (2015) Adjuvant denosumab in breast cancer (ABCSG-18): a multicentre, randomised, double-blind, placebo-controlled trial. Lancet 386:433-443

8. Smith MR, Saad F, Coleman R et al (2012) Denosumab and bone-metastasis-free survival in men with castration-resistant prostate cancer: results of a phase 3, randomised, placebo-controlled trial. Lancet 379:39-46

9. Suzuki Y, Nawata H, Soen S et al (2014) Guidelines on the management and treatment of glucocorticoidinduced osteoporosis of the Japanese Society for Bone and Mineral Research: 2014 update. J Bone Miner 
Metab 32:337-350

10. Kumamoto K, Nakamura T, Suzuki T et al (2010) Validation of the Japanese osteoporosis quality of life questionnaire. J Bone Miner Metab 28:1-7

11. Fukunaga M, Nakamura T, Shiraki M et al (2004) Absolute height reduction and percent height ratio of the vertebral body in incident fracture in Japanese women. J Bone Miner Metab 22:104-110

12. El Maghraoui A, Roux C (2008) DXA scanning in clinical practice. QJM 101:605-617

13. Kanis JA, Johnell O, Oden A et al (2000) Risk of hip fracture according to the World Health Organization criteria for osteopenia and osteoporosis. Bone 27:585-590

14. Kanis JA, Oden A, Johnell O et al (2001) The burden of osteoporotic fractures: a method for setting intervention thresholds. Osteoporos Int 12:417-427

15. Kanis JA (2002) Diagnosis of osteoporosis and assessment of fracture risk. Lancet 359:1929-1936

16. Johnell O, Kanis JA, Oden A et al (2005) Predictive value of BMD for hip and other fractures. J Bone Miner Res 20:1185-1194

17. Kanis JA, Seeman E, Johnell O et al (2005) The perspective of the International Osteoporosis Foundation on the official positions of the International Society for Clinical Densitometry. Osteoporos Int 16:456-459, discussion $579-80$

18. Arabi A, Baddoura R, Awada $\mathrm{H}$ et al (2007) Discriminative ability of dual-energy X-ray absorptiometry site selection in identifying patients with osteoporotic fractures. Bone 40:1060-1065

19. Leslie WD, Martineau P, Bryanton M, Lix LM (2019) Which is the preferred site for bone mineral density monitoring as an indicator of treatment-related anti-fracture effect in routine clinical practice? A registrybased cohort study. Osteoporos Int 30:1445-1453

20. Brown JP, Prince RL, Deal C et al (2009) Comparison of the effect of denosumab and alendronate on BMD and biochemical markers of bone turnover in postmenopausal women with low bone mass: a randomized, blinded, phase 3 trial. J Bone Miner Res 24:153-161

21. Saag KG, Wagman RB, Geusens $P$ et al (2018) Denosumab versus risedronate in glucocorticoid-induced osteoporosis: a multicentre, randomised, double-blind, active-controlled, double-dummy, non-inferiority study. Lancet Diabetes Endocrinol 6:445-454

22. Anastasilakis AD, Polyzos SA, Makras P, Aubry-Rozier B, Kaouri S, Lamy O (2017) Clinical features of 24 patients with rebound-associated vertebral fractures after denosumab discontinuation: systematic review and additional cases. J Bone Miner Res 32:1291-1296

23. Aubry-Rozier B, Gonzalez-Rodriguez E, Stoll D, Lamy O (2016) Severe spontaneous vertebral fractures after denosumab discontinuation: three case reports. Osteoporos Int 27:1923-1925

24. Cummings SR, Ferrari S, Eastell R et al (2018) Vertebral fractures after discontinuation of denosumab: a post hoc analysis of the randomized placebo-controlled FREEDOM trial and its extension. J Bone Miner Res 33:190-198

25. Fernández Fernández E, Benavent Núñez D, Bonilla Hernán G et al (2020) Multiple vertebral fractures following discontinuation of denosumab treatment: Ten clinical cases report. Reumatol Clin 16:480-484

26. Lamy O, Stoll D, Aubry-Rozier B, Rodriguez EG (2019) Stopping Denosumab. Curr Osteoporos Rep 17:8-15 
27. Anagnostis P, Paschou SA, Gonzalez-Rodriguez E et al (2019) Spontaneous vertebral fractures in males with osteoporosis after denosumab discontinuation: a report of two cases. J Clin Rheumatol. doi:10.1097/RHU.0000000000000979

28. Bone HG, Bolognese MA, Yuen CK et al (2011) Effects of denosumab treatment and discontinuation on bone mineral density and bone turnover markers in postmenopausal women with low bone mass. J Clin Endocrinol Metab 96:972-980

29. Lin T, Wang C, Cai XZ et al (2012) Comparison of clinical efficacy and safety between denosumab and alendronate in postmenopausal women with osteoporosis: a meta-analysis. Int J Clin Pract 66:399-408

30. Migliore A, Broccoli S, Massafra U, Cassol M, Frediani B (2013) Ranking antireabsorptive agents to prevent vertebral fractures in postmenopausal osteoporosis by mixed treatment comparison meta-analysis. Eur Rev Med Pharmacol Sci 17:658-667

\section{Figures}




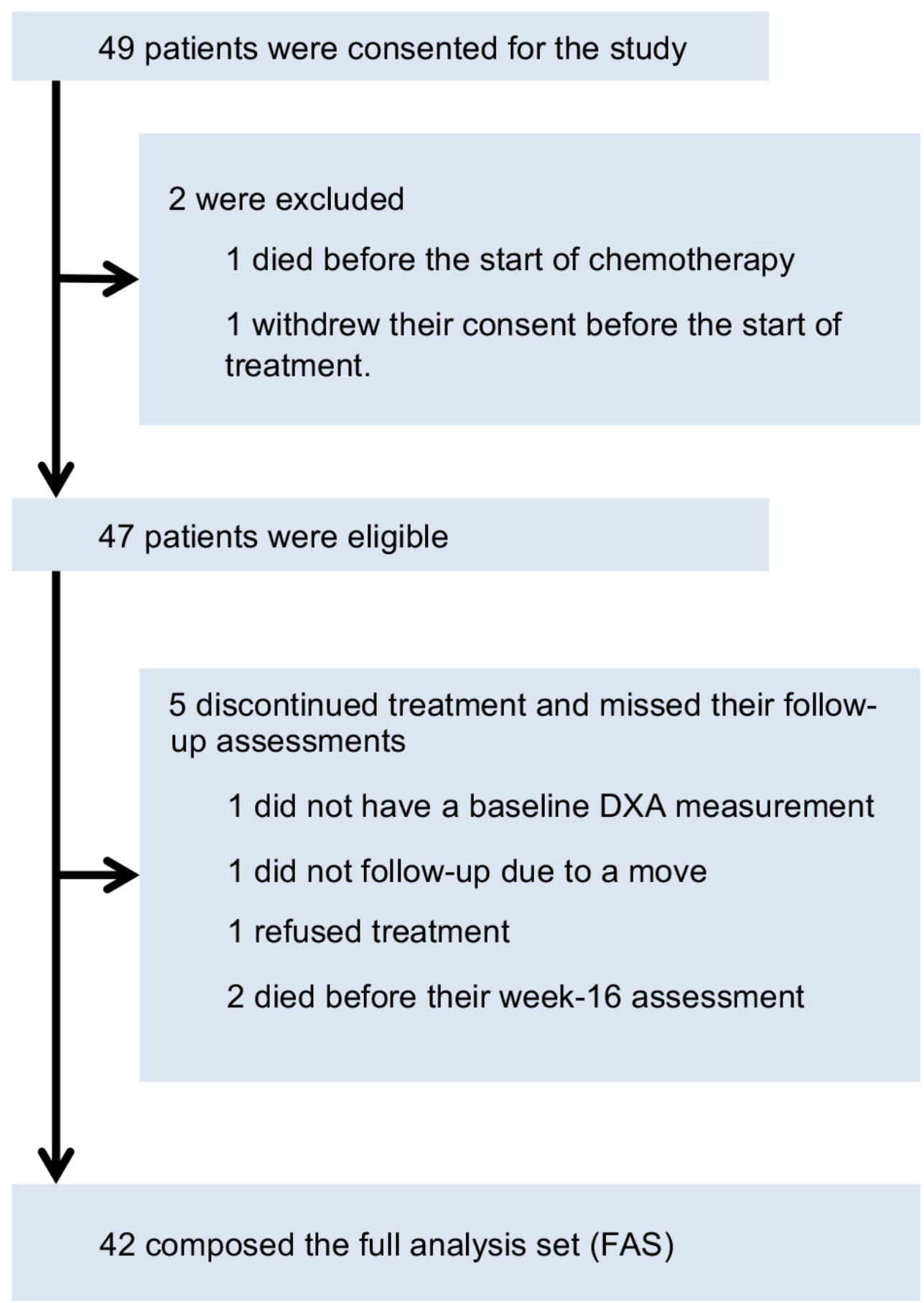

Figure 1

The full analysis set for assessing primary outcome therefore included 42 patients (male, 18; female, 24; age, 50-81 years; median age, 68 years) 
A

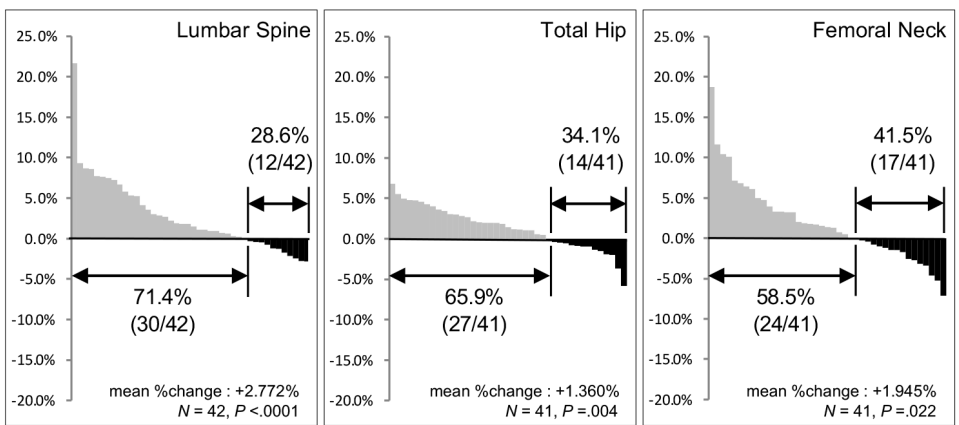

B

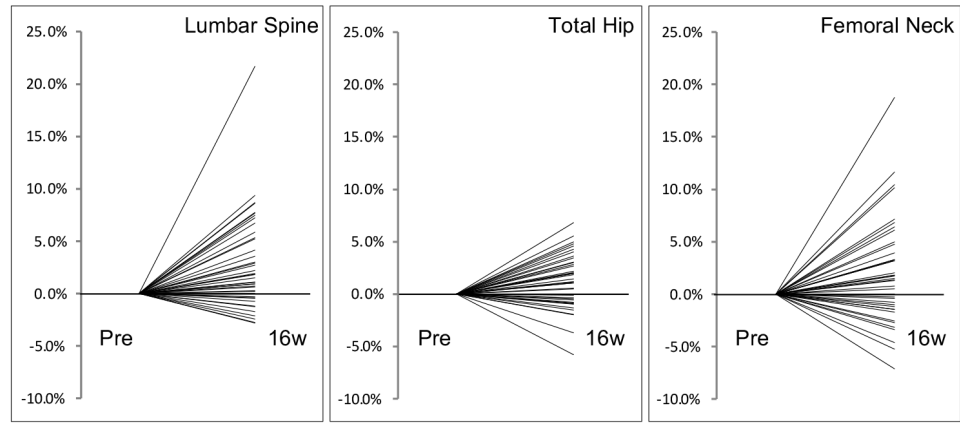

C
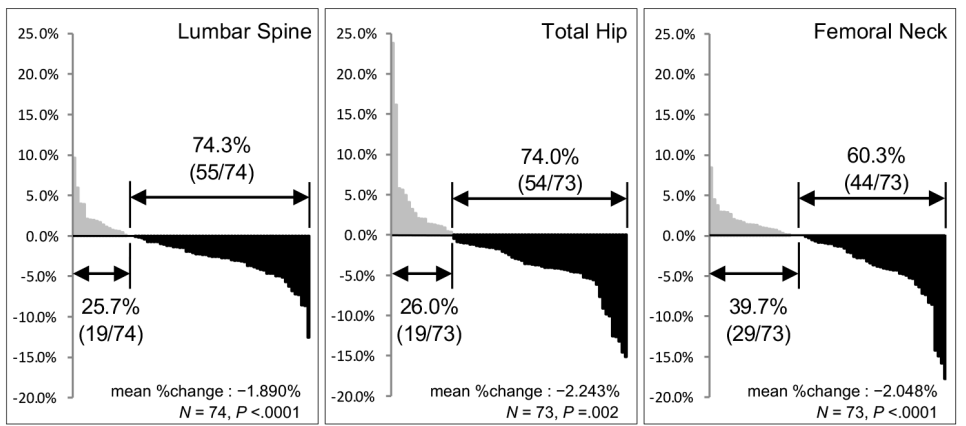

D

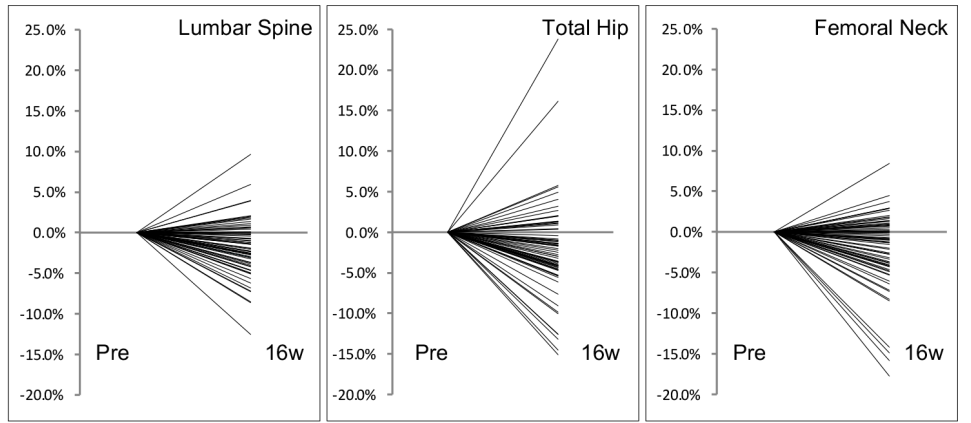

Figure 2

Mean BMD percent changes at 16 weeks were $2.772 \%$ [ $\mathrm{N}=42$ : $95 \%$ confidence interval $(\mathrm{Cl}), 1.350$ to $4.195 \%$, $1.360 \%(\mathrm{~N}=41: 95 \% \mathrm{Cl}, 0.526$ to $2.194 \%)$, and $1.945 \%(\mathrm{~N}=41: 95 \% \mathrm{Cl}, 0.375$ to $3.516 \%)$ in the $\mathrm{LS}, \mathrm{TH}$, and $\mathrm{FN}$, respectively (Table 2). Increased LS BMDs were observed in 30 patients (71.4\%), 27 (65.9\%) in the TH, and 24 (58.5\%) in the FN (Figure 2).

\section{Supplementary Files}

This is a list of supplementary files associated with this preprint. Click to download.

- SM1.docx

- SM2.docx 